\title{
Editorial
}

\section{Stingless bees: biology and management}

Social bees have been attracting scientific attention at least since Darwin struggled to fit sterile honeybee workers into his theory of evolution. More recently, the discovery of the dance communication by Karl von Frisch triggered a wave of interest in cooperation and later also in conflict in bee societies. Most of these studies have been carried out by scientists investigating temperate honeybees or bumblebees. This special issue is the first collection of papers devoted exclusively to the largest group of eusocial bees: the tropical stingless bees. These bees have traditionally received little attention probably as a result of their less accessible tropical habitats. Over the last 25 years, however, the number of scientific papers on stingless bees has been growing steadily both due to tropical scientists publishing in international journals and to cooperation between scientists from temperate and tropical climate areas. This issue strongly reflects both of these aspects.

Stingless bees are a relatively old lineage and the species vary widely in their physiology, behaviour, social organization and ecology. This makes them excellent subjects for comparative studies. They also have a long history in human culture both as providers of honey and wax (or cerumen) and as pollinators of our crops. This issue aims to present the state of the art in stingless bee biology and in the management of stingless bees. Comparative papers deal with nesting biology, community ecology, caste determination, sex-ratio allocation, division of labour and exocrine glands, whereas another paper presents a unique case of polygyny in social bees. Next, recent advances made by applying molecular techniques to stingless bee biology are presented. The final two papers provide updates on the use of stingless bees in applied pollination, both in the tropics and in temperate greenhouses, and on the state of meliponiculture, the keeping of stingless bees, around the world.

This series of papers, together with recent Apidologie reviews (issues 2/2004 and 2/2005) on stingless bee food source communication, social information flow, and male behaviour, and general reviews on multiple mating and conflict resolution in stingless bees, illustrate the state of the art in stingless bee research, in particular and social bee biology, in general. The papers also show that our knowledge on various topics of stingless bee biology and ecology still lags behind our knowledge of honeybees and bumblebees. Therefore, we hope that this issue stimulates future research and cooperation that will lead to an improved understanding of these bees, successful conservation of their threatened tropical habitats, and sustainable management of these important pollinators of crops and wild plants.

\section{Les abeilles sans aiguillon : biologie et gestion}

Les abeilles sociales ont attiré l'attention des scientifiques au moins depuis que Darwin a lutté pour faire entrer les ouvrières stériles de l'Abeille domestique dans sa théorie de l'évolution. Plus récemment la découverte par Karl von Frisch de la communication par les danses a suscité une vague d'intérêt pour la coopération puis plus tard pour les conflits dans les sociétés d'abeilles. La plupart de ces études ont été menées par des scientifiques étudiant abeilles domestiques et bourdons des régions tempérées. Ce numéro spécial rassemble pour la première fois des articles exclusivement consacrés au plus grand groupe d'abeilles eusociales : les abeilles sans aiguillon tropicales. Par tradition ces abeilles ont reçu peu d'attention probablement parce que leurs habitats tropicaux sont moins accessibles. Pourtant au cours des 25 dernières années le nombre d'articles sur les abeilles sans aiguillon n'a cessé de croître régulièrement, à la fois parce que les 
scientifiques des régions tropicales ont publié dans les revues internationales et parce qu'ils ont coopéré avec leurs collègues des zones tempérées. Ce numéro reflète ces deux aspects.

Les abeilles sans aiguillon sont d'une lignée relativement ancienne et la physiologie, le comportement, l'organisation sociale et l'écologie sont très variables d'une espèce à l'autre. Cela en fait d'excellents sujets pour des études comparatives. Elles ont aussi une longue histoire dans la culture humaine à la fois comme fournisseurs de miel et de cire (ou cerumen) et comme pollinisateurs des cultures. Ce numéro souhaite présenter l'état d'avancement des connaissances dans les domaines de la biologie et de la gestion des abeilles sans aiguillon. Des articles comparatifs traitent de la biologie de la nidification, de l'écologie des communautés, de la détermination des castes, de la répartition du sex ratio, de la division du travail et des glandes exocrines, tandis qu'un autre article présente l'unique cas de polygynie chez les abeilles sociales. Sont aussi présentés les progrès récents obtenus par l'utilisation des techniques moléculaires. Les deux derniers articles fournissent une mise à jour de l'utilisation des abeilles sans aiguillon dans la pollinisation appliquée, sous les tropiques comme dans les serres des pays tempérés, et de l'état de la méliponiculture, ou élevage des abeilles sans aiguillon, dans le monde.

Cette série d'articles, avec les articles de synthèse parus récemment dans Apidologie $\left(n^{\circ} 2 / 2004\right.$ et 2/2005) sur la communication de la source de nourriture, le flux d'information sociale, le comportement des mâles, ainsi que les articles de synthèse généraux sur l'accouplement multiple et la résolution des conflits chez les abeilles sans aiguillon, illustrent l'état d'avancement des recherches sur ces abeilles en particulier et sur la biologie des abeilles sociales en général. Ces articles montrent aussi que notre connaissance sur les divers thèmes de la biologie et l'écologie des abeilles sans aiguillon est à la traîne comparée à nos connaissances sur les abeilles domestiques et les bourdons. Nous espérons donc que ce numéro spécial stimulera la recherche et les coopérations futures et que cela conduira à une meilleure compréhension de ces abeilles, à une préservation réussie des habitats tropicaux menacés et à une gestion durable de ces pollinisateurs importants pour les cultures et les plantes sauvages.

\section{Stachellose Bienen: Biologie und Management}

Soziale Bienen haben spätestens seit Darwin Wissenschaftler angezogen, die sich damit beschäftigten das Phänomen der sterilen Arbeiterinnen der Honigbiene mit der Evolutionstheorie zu vereinbaren. Mit der Entdeckung der Tanzsprache der Bienen durch Karl von Frisch wurde dann auch das Interesse zunächst für die Kooperation bei Bienenvölkern und später dann auch für die Frage der Konflikte geweckt. Die meisten dieser Untersuchungen wurden an den in gemässigten Klimaten vorkommenden Honigbienen und Hummeln durchgeführt. Dieses Sonderheft widmet sich nun exklusiv der grössten Gruppe der eusozialen Bienen, den tropischen Stachellosen Bienen. Diese Bienen haben in der Vergangenheit weniger Aufmerksamkeit gefunden, vermutlich aufgrund des schwierigeren Zugangs zu tropischen Habitaten. Während der letzten 25 Jahre hat jedoch die Zahl der wissenschaftlichen Veröffentlichungen über Stachellose Bienen stetig zugenommen, da zum einen immer mehr Wissenschaftler aus Tropenländern in internationalen Zeitschriften publizieren und zum anderen weil die internationalen Kooperationen zwischen Wissenschaftlern zunehmen. Das Sonderheft macht gerade auch diese Aspekte deutlich.

Stachellose Bienen sind eine phylogenetisch relativ alte Linie und die einzelnen Arten variieren dementsprechend stark hinsichtlich ihrer Physiologie, ihres Verhaltens, ihrer sozialen Organisation und Ökologie. Dies macht sie zu exzellenten Untersuchungsobjekten für vergleichende Studien. Auch im kulturellen Bereich haben sie eine lange Geschichte, sowohl als Lieferanten für Honig, Wachs (oder Cerumen) und als Bestäuber von Kulturpflanzen. Diese Sonderheft präsentiert den aktuellen Forschungsstand in der Biologie der Stachellosen Bienen und in ihrer Haltung. Mehrere Manuskripte betrachten sie vergleichend hinsichtlich der Nestbiologie, Ökologie von Bienengemeinschaften, Kastendetermination, Allokationen im Geschlechtstierverhältnis, 
Arbeitsteilung und Struktur exokriner Drüsen. Andere Arbeiten beleuchten einen für soziale Bienen besonderen und einzigartigen Aspekt, die bei einer Art vorkommende Polygynie. Ein weiteres Manuskript umreisst die Bedeutung molekularer Techniken für Arbeiten mit Stachellosen Bienen. Die letzten beiden Manuskripte stellen den neuesten Stand in der Nutzung Stachelloser Bienen als angewandte Bestäuber in den Tropen und auch in Gewächshäusern dar und geben ein umfassendes Bild zur Meliponikultur rund um die Welt.

Diese Serie von Arbeiten, zusammen mit anderen in den letzten Jahren in der Apidologie veröffentlichten Übersichtsartikeln (in den Heften 02/2004 und 02/2005) über Kommunikation von Futterquellen, dem Fluss sozialer Information, dem Verhalten von Männchen, sowie allgemeine Übersichtsartikel zur Paarungsbiologie und Lösungsstrategien in sozialen Konflikte, beleuchten den aktuellen Wissensstand der Forschung über Stachellose Bienen und zur Biologie sozialer Bienen im allgemeinen. Diese Arbeiten zeigen auch, dass unser Wissen in verschiedenen Bereichen der Biologie und Ökologie der Stachellosen Bienen noch weit hinter dem Wissen über Honigbienen und Hummeln hinterherhinkt. Wir hoffen deshalb, dass dieses Sonderheft stimulierend wirkt auf künftige Forschungs- und Kooperationsprojekte und so zu einem vertieften Verständnis dieser Bienen, zum erfolreichen Schutz ihrer tropischen Habitate und zur nachhaltigen Nutzung dieser wichtigen Bestäuber von Nutz- und Wildpflanzen führen wird.

Jacobus C. Biesmeijer Klaus Hartfelder

Vera Lucia Imperatriz-Fonseca February 2006 